Revista EIA, ISSN 1794-1237 Número 8, p. 165-180. Diciembre 2007

Escuela de Ingeniería de Antioquia, Medellín (Colombia)

\title{
EVALUACIÓN DEL RIESGO DE CONTAMINACIÓN POR METAMIDOFOS EN LA MICROCUENCA EL SALTO DEL MUNICIPIO DE EL SANTUARIO, ANTIOQUIA
}

\author{
David Horacio RamíreZ* \\ Adriana Marcela Zuluaga* \\ Evelio DE JESÚS GÓMEZ**
}

\section{RESUMEN}

En este trabajo de investigación, que se desarrolló en la microcuenca El Salto del municipio de El Santuario, se estimó la presencia del Metamidofos, en plantas de repollo (Brassica oleraceae) y en el suelo y su riesgo de contaminación en aguas para el consumo humano. En el repollo el nivel de concentración del Metamidofos sobrepasó los límites máximos permisibles establecidos para esta planta por el Codex Alimentarius; en el suelo no se encontraron residuos del producto, pero sí en el agua de escorrentía y en dos sitios del cauce de la quebrada El Salto localizados aguas arriba de la bocatoma del acueducto municipal; no se detectó Metamidofos en la muestra de agua de la bocatoma del acueducto ni en la red de distribución del acueducto municipal.

En la estimación cualitativa del riesgo de contaminación se utilizaron como variables de vulnerabilidad y amenaza la precipitación, la escorrentía, el coeficiente de escorrentía, el tiempo de concentración, la erosión, algunas propiedades físicas, químicas y biológicas del suelo y la pendiente de los terrenos. Se observó que el riesgo se presenta por la alta cantidad de insecticida utilizado y su frecuencia de utilización, que se incrementa según la distribución e intensidad de las lluvias y del potencial de erosión y este, a su vez, por el uso y manejo no adecuado del suelo. En la parcela experimental y en un terreno aledaño sembrado con pasto kikuyo (Pennisetum clandestinum), se calculó la población por unidad de área de la lombriz de tierra (Lumbricus terrestris). La ausencia de este organismo en la parcela cultivada con repollo (Brassica oleraceae) se puede considerar como indicador biológico por la contaminación de este insecticida en el suelo.

PALABRAS CLAVE: Metamidofos; contaminación por plaguicidas; repollo; Brassica oleraceae; microcuenca El Salto.

* Ingeniero Ambiental. Universidad Católica de Oriente.ramirezmaciad@gmail.com; azuluaga1@gmail.com

** Ingeniero Agrónomo, Universidad Nacional de Colombia. Docente, Universidad de Antioquia. gadan@une.net.co 


\section{ABSTRACT}

This investigation was done in El Salto watershed basin located in the municipality of El Santuario. It was examined the presence of Metamidofos in plants of cabbage, in soils, with risk of contamination in waters for the human consumption. The level of concentration of Metamidofos exceeded the maximum limits established by Codex Alimentarius. In the soil, remainders of the product were not found, but they were present in runoff and in two sites of the channel of the gorge, located upstream of the intake of the municipal water system. Metamidofos was not found in the water sample of intake nor in the distribution network of the municipal water system.

In the qualitative estimation of the contamination risk, precipitation, runoff, coefficient of runoff, time of concentration, physical and chemical characteristics, erosion, some properties and the slope of lands were chosen as variable of vulnerability and threat; it was observed that the risk appears by the high amount of insecticide used and its frequency of use which is increased by the distribution and intensity of rains, the erosion potencial that occurs by inappropiate use of the ground. In the experimental parcel and a near land cultivated with grass Kikuyo (Pennisetum clandestinum) was considered the population by area unit of the earthworm (Lumbricus terrestris). The absence of this organism in the parcel cultivated with cabbage can be a biological factor by the contamination of this insecticide in the ground.

KEY WORDS: Metamidofos; contamination by plaguicides; cabbage; Brassica oleraceae; El Salto watershed basin.

\section{INTRODUCCIÓN}

El Oriente Antioqueño (región de altiplano) es considerado en el país como una de las principales zonas de producción, con énfasis en productos agrícolas de tierra fría y desarrollada en unidades de producción subfamiliar. Allí se cultivan, entre otros productos, papa, fríjol, maíz, hortalizas (repollo, zanahoria, lechuga, coliflor, pimentón), tomate, arveja, habichuela y frutales de clima frío moderado (mora de Castilla, aguacate, fresa, tomate de árbol). Según Galeano et al. (2001), como control fitosanitario, se utilizan más de $15 \mathrm{~kg}$ de fungicidas y $10 \mathrm{~L}$ de insecticidas por hectárea por cosecha, además los agricultores sobredosifican estos productos, no calibran los equipos de aspersión y no tienen precaución en la manipulación y aplicación; ello da como resultado la posible contaminación de suelos, aire y aguas que abastecen acueductos veredales y municipales y en del producto de sus cosechas.

Con el interés de conocer lo expuesto, se evaluó el riesgo de la contaminación por Metamidofos en el agua de la microcuenca El Salto que abastece el acueducto urbano del municipio de El Santuario, el cual, según Giraldo et al. (2002), es considerado como uno de los municipios de Antioquia donde se presentan los mayores índices de consumo de plaguicidas. Para ello, se consideró la concentración del producto en el agua, suelo y material vegetal, identificando variables como la precipitación, escorrentía, propiedades internas y externas del suelo, erosión, pendientes de los cultivos y otras variables que permitan evaluar si existe el riesgo de contaminación por este producto. Si se constata de manera tangible la presencia de residuos en estos productos, será necesario plantear 
programas de educación al agricultor para hacerlo consciente del riesgo para la salud humana, así como para capacitarlo en la aplicación adecuada de plaguicidas y en el manejo y conservación de suelos para producción limpia.

\section{GENERALIDADES}

\subsection{El agua lluvia como factor de transporte de materiales}

La precipitación se define como la cantidad de agua, tanto en forma líquida (lluvia) como sólida, que cae a la superficie de la Tierra con una velocidad apreciable y en una unidad de área. Esta se expresa como lámina de agua ( $\mathrm{mm}$ ), que a la vez corresponde a la relación entre el volumen de agua que cae en un área específica de la superficie terrestre, relación que se expresa en $\mathrm{L} / \mathrm{m}^{2}$.

Se tiene que la erosión por escorrentía o escurrimiento (E) se presenta cuando el agua de lluvia que no alcanza a infiltrarse circula por la superficie del terreno, arrastrando el suelo desprendido por la lluvia y a la vez eroda las zonas del terreno por la cual se mueve. En forma estricta, se expresa como la relación entre el agua que escurre $(\mathrm{Ae})$ y el área (Ua) donde se presenta su movimiento:

$$
E=A e / U a
$$

Donde:

$$
\begin{array}{ll}
\text { E: } & \text { Escorrentía }\left(\mathrm{L} / \mathrm{m}^{2}\right) \text { o }(\mathrm{mm}) \\
\text { Ae: } & \text { Cantidad de agua que escurre }(\mathrm{L}) \\
\text { Ua: } & \text { Unidad de área }\left(\mathrm{m}^{2}\right)
\end{array}
$$

Con ella, se obtiene el coeficiente de escorrentía (Ce) que resulta de la relación entre la escorrentía (E) y la lámina de lluvia dada como precipitación (Pp) y se expresa como:

$$
\mathrm{Ce}=\mathrm{E} / \mathrm{Pp}
$$

Donde:

Ce: Coeficiente de escorrentía. (Puede ser expresado en \%).
E: Escorrentía $\left(\mathrm{L} / \mathrm{m}^{2}\right)$ o $(\mathrm{mm})$

Pp: Precipitación (mm)

\subsection{Caracteristicas del Metamidofos}

Lozano (2001) afirma que el Metamidofos se degrada al aire libre en sistemas naturales de agua, tiene una vida media de 15,9 días en el agua y de 7,7 días en el sedimento. El Metamidofos en el suelo es poco persistente y es altamente móvil, ya que es absorbido por el suelo en pequeñas cantidades; dada la rapidez de la degradación de la sustancia y alta movilidad, se lixivia en las capas del suelo.

En el agua y en el sedimento es más persistente:

Con $\mathrm{pH}$ de 5 tiene una vida media de 309 días.

Con $\mathrm{pH}$ de 7 tiene una vida media de 27 días.

Con $\mathrm{pH}$ de 3 tiene una vida media de 3 días.

\subsection{Persistencia y bioconcentración}

La persistencia de un plaguicida se refiere al periodo de tiempo que permanece biológicamente activo y sigue siendo tóxico para los efectos que fue aplicado (Arango, 1996). En general, los plaguicidas persistentes (los que permanecen biológicamente activos por largos periodos) son menos solubles y volátiles, pero tienen una fuerte tendencia a ser absorbidos (enlazados a partículas de suelo). Cuando un plaguicida no es metabolizado o excretado por un organismo, por lo general, se almacena en los tejidos grasos. Los últimos organismos de la cadena alimenticia tienden a tener mayores concentraciones de plaguicidas almacenados en sus cuerpos que los de los niveles inferiores, tendencia que es conocida como bioconcentración ("magnificación" biológica). Al respecto, Cornare (2003) afirma que la persistencia de los plaguicidas sintéticos se debe a que su estructura química es nueva y los organismos descomponedores naturales, como las bacterias, no tienen vías para degradarlos; por consiguiente, se acumulan en el ambiente y en la cadena alimenticia. 


\section{MATERIALES Y MÉTODOS}

Como área de estudio se consideró la microcuenca El Salto, ubicada en el municipio de El Santuario, Antioquia, la cual surte el acueducto para el área municipal. Hace parte de la subcuenca La Marinilla y esta a su vez hace parte de la cuenca río Negro-Nare, perteneciente a la gran cuenca del río Magdalena. La microcuenca presenta los siguientes indicadores morfométricos: área aproximada de $3,37 \mathrm{~km}^{2}$, elevaciones sobre el nivel del mar entre $2450 \mathrm{~m}$ y $2116 \mathrm{~m}$, temperatura promedio anual de $13,3^{\circ} \mathrm{C}$, pendiente media del cauce $8,33 \%$, pendiente media de la cuenca de $36,02 \%$ y una densidad de drenaje de $5,65 \mathrm{~km} / \mathrm{km}^{2}$; el suelo se ha desarrollado a partir de cenizas volcánicas depositadas sobre granodioritas y cuarzodioritas del Batolito Antioqueño, originando materiales heterogéneos y heterométricos (Proingecon, 2000).

La implementación de la parcela de escorrentía se hizo para dos periodos de lluvia denominados periodos de alta y baja frecuencia de lluvias. Para el periodo de alta frecuencia de lluvias la parcela tenía forma ovalada con dimensión mayor en la dirección de la pendiente del terreno, de un área aproximada de $633 \mathrm{~m}^{2}$ y tenía un cultivo de repollo (Brassica oleracea $L$ ) con un mes de edad de establecido, sembrado en surcos, con pendiente aproximada de $22 \%$. Para el periodo de baja frecuencia de lluvias se utilizó un terreno aledaño a la parcela anterior, con iguales características físicas, químicas y fisiográficas, solo que su área corresponde a $500 \mathrm{~m}^{2}$ y presentó un cultivo de remolacha (Beta vulgaris L.) de 15 días de establecido.

Cada parcela en su periodo descargó su flujo en un canal central, al final del cual se realizó una excavación con dimensiones laterales de $0,87 \mathrm{~m}$ por 1,00 $\mathrm{m}$ y con profundidad $0,438 \mathrm{~m}$ (capacidad aproximada de $380 \mathrm{~L}=0,38 \mathrm{~m}^{3}$ ), su fondo y paredes laterales se recubrieron con un plástico negro de calibre 12, lo que permitió la acumulación diaria del agua y el suelo que transporta la lámina de escorrentía. Esta excavación se convierte en lo que se denomina como tanque de escorrentía.

Para medir la precipitación del área se instaló dentro de la parcela experimental un pluviómetro tipo embudo-botella. Todas las mañanas se midió la cantidad de agua (litros) que contenía la botella. Después de la medición se botó el agua del tubo y se reinstaló el pluviómetro para el siguiente registro.

La precipitación $(\mathrm{Pp})$ en $\mathrm{mm}$ se obtiene de la expresión:

$$
P p=\frac{\text { Volumen }}{\text { Area }}=\frac{L}{m^{2}}=m m
$$

La información del pluviómetro se tabuló, teniendo en cuenta los dos periodos evaluados, denominados de baja y alta frecuencia de lluvias.

Las variables ambientales evaluadas en las parcelas de escorrentía fueron: humedad y porosidad del suelo, escorrentía, coeficiente de escorrentía, tiempo de concentración, índice de forma y suelo perdido por unidad de área.

\subsection{Estimación de la presencia del Metamidofos}

Se tomaron muestras de suelos en los primeros $5 \mathrm{~cm}$ y debajo del área de influencia de cada planta muestreada, cuyo análisis en el Laboratorio Nacional de Insumos Agrícolas del ICA en Bogotá permitió estimar el contenido de organofosforados por el método de extracción por Soxhlet e identificación por cromatografía de gases con detector fotométrico de llama. Mediante un muestreo aleatorio estratificado, se tomaron hojas de la cabeza de plantas de repollo (Brassica oleracea $L$ ), se analizaron en el mismo laboratorio, donde se calculó el contenido de organofosforados por el mismo método. 
En fuentes de agua de la cuenca El Salto, en sitios definidos previamente (tanque de escorrentia; en el curso del agua, entre la parcela y la entrada del agua a la bocatoma y en la entrada de la bocatoma del acueducto municipal), se recolectaron muestras de agua ( $3 \mathrm{~L} /$ muestra), en los periodos de alta y baja frecuencia de lluvias respectivamente; y una muestra en la red de distribución del acueducto municipal. Su análisis se efectúo en el Centro de Servicios de Análisis de Aguas (Censa-Cornare), donde se estimó la presencia del Metamidofos mediante el cromatógrafo de gases con MSD, detector selectivo de masas, Hewlett Packard 6890/597A, en columna HP-5MS, flujo en helio $1,0 \mathrm{~mL} / \mathrm{min}$, con temperaturas: detector $280^{\circ} \mathrm{C}$, inyección $250^{\circ} \mathrm{C}$ y columna $100-280^{\circ} \mathrm{C}$.

Como indicador biológico de contaminación, se consideró la lombriz de tierra (Lumbricus terrestris). Para ello, se realizó un muestreo poblacional en la parcela experimental que contiene repollo (Brassica oleracea $L$ ) y en un lote aledaño con características de suelo muy similares a la parcela del ensayo, que al momento del muestreo presentaba pasto kikuyo (Pennisetum clandestinum L), al cual no se le ha aplicado plaguicidas y se dedica a pastoreo.

En cada parcela, se seleccionaron al azar tres puntos de muestreo, en cada uno se realizó una excavación de $50 \mathrm{~cm}$ x $50 \mathrm{~cm}$ y con una profundidad de $10 \mathrm{~cm}$. En el suelo obtenido de la excavación se realizó el conteo de organismos, teniendo en cuenta los diferentes estados de desarrollo.

\section{ESTIMACIÓN DEL RIESGO DE CONTAMINACIÓN DE LAS AGUAS POR EL METAMIDOFOS}

\section{El riesgo $(\mathbf{R})$}

Además de lo que establecen el Decreto 1843 de 1991 y Smith et al. (2005), los cuales consideran el riesgo (R) como el resultado esperado por las pérdidas o daños causados por un evento amenazante ante una determinada comunidad y presenta una función directa entre la amenaza (A) y la vulnerabilidad (V), lo cual se puede expresar como:

$$
R=A \times V
$$

Para el caso de los plaguicidas que se utilizan para el control de las plagas y enfermedades de los cultivos que prevalecen en una cuenca hidrográfica, el riesgo se presenta cuando los ingredientes activos de estos productos se hacen presentes en las aguas que se usan para el consumo humano, donde la magnitud del riesgo depende del grado de toxicidad del producto y de su concentración en el agua.

\subsection{Rango estimado del riesgo}

La categoría del riesgo da origen a la posible contaminación de las aguas para consumo humano y en especial la voz de alerta para establecer las estrategias para la mitigación de sus impactos sociales y ambientales. Para ello, se deben tener en cuenta las variables que presentan mayor relevancia en el riesgo que, en general, son de fácil manejo e implementación en los programas de extensión rural. Para el desarrollo de este componente, se utilizaron las variables de la propuesta para la evaluación del riesgo por plaguicidas en aguas para consumo humano desarrollada por Gómez (2006a).

\section{VALOR DE LA AMENAZA SEGÚN LOS CRITERIOS DE EVALUACIÓN DEL PLAGUICIDA}

\subsection{Según la toxicidad}

Con base en lo que establece el artículo 14 del Decreto 1843 de 1991, en lo relacionado con las categorías de toxicidad de los plaguicidas, se proponen los valores consignados en la tabla 1 . 
Tabla 1. Valor de la amenaza según categoría de toxicidad del plaguicida

\begin{tabular}{|c|c|c|}
\hline \multicolumn{2}{|c|}{ Categoría } & \multirow{2}{*}{$\begin{array}{c}\text { Valor como } \\
\text { Amenaza }\end{array}$} \\
\cline { 1 - 2 } Número & Toxicidad & 10 \\
\hline I & Extremadamente tóxicos & 8 \\
\hline III & Altamente tóxicos & 6 \\
\hline IV & Ligeramente tóxicos & 4 \\
\hline V & No tóxico & 2 \\
\hline
\end{tabular}

\subsection{Según la solubilidad}

Para la propuesta es de gran relevancia la solubilidad del plaguicida y ante todo lo relacionado con el agua (tabla 2).

Tabla 2. Valor de la amenaza según el medio de la solubilidad del plaguicida

\begin{tabular}{|c|c|}
\hline Solubilidad en & Valor como amenaza \\
\hline Agua & 10 \\
\hline Solventes & 5 \\
\hline
\end{tabular}

\subsection{Parámetros de evaluación del agua lluvia}

Para esto se debe tener en cuenta la suma de los valores de las tablas correspondientes a la precipitación media anual, intensidad, frecuencia y duración de las lluvias.

La precipitación tiene un papel aun más importante en lo que se refiere al transporte de material erosionado. Desde el momento en el que cualquier lugar reciba más agua (en forma de lluvia, nieve derretida o hielo) de la que el terreno pueda absorber, el excedente fluirá hacia niveles más bajos arrastrando el material suelto. Las laderas sufren una erosión laminar, en la cual la escorrentía arrastra la capa superior del suelo, en algunos casos, sin dejar rastros visibles de haber erosionado esa superficie.
Con base en el sistema de zonas de vida de Colombia, (Espinal, 1977), se proponen los valores de amenaza por la precipitación media anual de la zona (tabla 3).

\subsection{Frecuencia de los aguaceros}

Esta hace alusión al periodo seco entre aguaceros consecutivos. A menos días secos entre aguaceros, mayor es la cantidad de agua de lluvia en el periodo; ello origina los periodos de alta precipitación. Cuando el contenido de agua en el suelo sobrepasa el potencial de humedad con capacidad de campo, se incrementa el potencial de transporte de las aguas (tabla 4)

\subsection{Duración de los aguaceros}

Se considera el tiempo que transcurre mientras se presenta una lluvia. Para la propuesta, implica que los aguaceros de corta duración pueden tener una mayor incidencia en el transporte de contaminantes en una cuenca hidrográfica, aspecto que a la vez está relacionado con la intensidad media de los aguaceros de la zona (tabla 5).

\section{VULNERABILIDAD SEGÚN LAS CARACTERÍSTICAS FÍSICAS DE LA CUENCA HIDROGRÁFICA}

\section{1 Índice de forma (If)}

Según Espejo (1985) e Ingeominas (2002), este índice compara la microcuenca con una circunferencia cuyo círculo inscrito tiene la misma superficie de la microcuenca, donde un valor igual o cercano a la unidad correspondería a una microcuenca hipotética cuadrada o circular e indica que la cuenca tiene tendencia a concentrar sus aguas en un tiempo reducido. Cuando se aleja de la unidad se puede interpretar que la microcuenca 
Tabla 3. Evaluación de la precipitación media anual como potencial de amenaza en la contaminación por plaguicidas en una cuenca hidrográfica

\begin{tabular}{|c|c|c|c|}
\hline \multicolumn{2}{|l|}{ Zona de vida } & \multirow{2}{*}{$\frac{\text { Precipitación media anual }}{\mathrm{mm}}$} & \multirow{2}{*}{$\begin{array}{l}\text { Valor como } \\
\text { amenaza }\end{array}$} \\
\hline Nombre & Símbolo & & \\
\hline Matorral desértico tropical & $m d-T$ & $125-250$ & 1 \\
\hline Monte espinoso tropical & $m e-T$ & $250-500$ & 2 \\
\hline Bosque muy seco tropical & $\mathrm{bms}-\mathrm{T}$ & $500-1000$ & 3 \\
\hline Bosque seco tropical & bs $-T$ & $1000-2000$ & 4 \\
\hline Bosque húmedo tropical & $\mathrm{bh}-\mathrm{T}$ & $2000-4000$ & 6 \\
\hline Bosque muy húmedo tropical & $\mathrm{bmh}-\mathrm{T}$ & $4000-8000$ & 8 \\
\hline Bosque pluvial tropical & $\mathrm{bp}-\mathrm{T}$ & $>8000$ & 10 \\
\hline Monte espinoso premontano & me - PM & $250-500$ & 2 \\
\hline Bosque seco premontano & bs - PM & $500-1000$ & 4 \\
\hline Bosque húmedo premontano & bh - PM & $1000-2000$ & 6 \\
\hline Bosque muy húmedo premontano & bmh - PM & $2000-4000$ & 8 \\
\hline Bosque pluvial premontano & $\mathrm{bp}-\mathrm{PM}$ & $>4000$ & 10 \\
\hline Bosque seco montano bajo & bs - MB & $500-1000$ & 4 \\
\hline Bosque húmedo montano bajo & bh - MB & $1000-2000$ & 6 \\
\hline Bosque muy húmedo montano bajo & bmh - MB & $2000-4000$ & 8 \\
\hline Bosque pluvial montano bajo & $\mathrm{bp}-\mathrm{MB}$ & $>4000$ & 10 \\
\hline Bosque húmedo montano & bh $-M$ & $500-1000$ & 6 \\
\hline Bosque muy húmedo montano & $\mathrm{bmh}-\mathrm{M}$ & $1000-2000$ & 8 \\
\hline Bosque pluvial montano & $\mathrm{bp}-\mathrm{M}$ & $>2000$ & 10 \\
\hline Páramo subalpino & $p-S A$ & $>500$ & 8 \\
\hline Páramo pluvial subalpino & $\mathrm{pp}-\mathrm{SA}$ & $>500$ & 10 \\
\hline Tundra pluvial alpina & $t p-A$ & $>500$ & 10 \\
\hline Nival & $\mathrm{N}$ & - & 10 \\
\hline
\end{tabular}

Tabla 4. Evaluación de la frecuencia de la lluvia como potencial de amenaza en la contaminación por plaguicidas en una cuenca hidrográfica

\begin{tabular}{|c|c|c|}
\hline \multicolumn{2}{|c|}{ Frecuencia } & \multirow{2}{*}{$\begin{array}{c}\text { Valor como } \\
\text { amenaza }\end{array}$} \\
\hline Periodo seco & Denominación & \\
\hline 1 día & Muy alta & 10 \\
\hline 1-5 días & Alta & 8 \\
\hline 5-10 días & Moderada & 6 \\
\hline 1-30 días & Moderadamente baja & 4 \\
\hline 1-2 meses & Baja & 2 \\
\hline 2-6 meses & Muy baja & 1 \\
\hline
\end{tabular}

Tabla 5. Evaluación de la duración de la lluvia como potencial de amenaza en la contaminación por plaguicidas en una cuenca hidrográfica

\begin{tabular}{|c|c|c|}
\hline \multicolumn{2}{|c|}{ Duración } & \multirow{2}{*}{$\begin{array}{c}\text { Valor como } \\
\text { amenaza }\end{array}$} \\
\hline Tiempo & Denominación & 1 \\
\hline Menos de 1 Hora & Muy baja & 2 \\
\hline 1-2 horas & Baja & 4 \\
\hline 4-8 horas & $\begin{array}{c}\text { Moderadamente } \\
\text { baja }\end{array}$ & 6 \\
\hline 8-16 horas & Moderada & 8 \\
\hline Más de 16 horas & Muy alta & 10 \\
\hline
\end{tabular}


favorece la regulación hidrológica, presentándose un tiempo de concentración alto con escurrimientos demorados.

Tabla 6. Vulnerabilidad del índice de forma en la capacidad del transporte del contaminante

\begin{tabular}{|c|c|c|}
\hline \multicolumn{2}{|c|}{ Factor de forma (Ff) } & \multirow{2}{*}{$\begin{array}{c}\text { Valor como } \\
\text { vulnerabilidad }\end{array}$} \\
\cline { 1 - 2 } $\begin{array}{c}\text { Valor de la } \\
\text { relación }\end{array}$ & Forma de la cuenca & 10 \\
\hline Menor de 1 & Circular o cuadrada & 5 \\
\hline Mayor de 1 & Alargada & \\
\hline
\end{tabular}

\subsection{Tiempo de concentración}

Se considera el tiempo que tarda la lámina de agua en recorrer el espacio entre el divorcio de sus aguas al sitio de aforo (FAO, 1976).
Tabla 7. Vulnerabilidad del tiempo de concentración en función del transporte del contaminante

\begin{tabular}{|c|c|c|}
\hline \multicolumn{2}{|c|}{ Tiempo de concentración } & \multirow{2}{*}{$\begin{array}{c}\text { Valor como } \\
\text { vulnerabilidad }\end{array}$} \\
\cline { 1 - 2 } Horas & Descripción & 10 \\
\hline$<1$ & Muy bajo & 8 \\
\hline $1-12$ & Bajo & 8 \\
\hline $12-24$ & Moderadamente bajo & 4 \\
\hline $24-48$ & Alto & 2 \\
\hline$>48$ & Muy Alto & \\
\hline
\end{tabular}

\subsection{Profundidad efectiva del suelo}

Se entiende la profundidad a la cual se desarrollan las raíces sin impedimentos físicos o químicos. En la tabla 8, Mannering, citado por López (2000), propone una tolerancia a la pérdida de suelo por la erosión, según la profundidad efectiva del suelo y que es ajustada por el autor Gómez (2006).

Tabla 8. Tolerancia a la pérdida de suelo por la erosión, según la profundidad efectiva del suelo y su relación con la vulnerabilidad del suelo como regulador del agua que se podría liberar a las corrientes de una cuenca hidrográfica

\begin{tabular}{|c|c|c|c|}
\hline $\begin{array}{c}\text { Profundidad efectiva } \\
\mathrm{cm}\end{array}$ & Denominación & $\begin{array}{c}\text { Tolerancia a la pérdida de } \\
\text { suelo } \\
\text { t/ha }\end{array}$ & $\begin{array}{c}\text { Valor como } \\
\text { vulnerabilidad }\end{array}$ \\
\hline$<25$ & Muy superficial & $<4$ & 10 \\
\hline $26-50$ & Superficial & $5-8$ & 8 \\
\hline $51-100$ & Moderadamente profundo & $9-12$ & 6 \\
\hline $101-150$ & Profundo & $13-16$ & 4 \\
\hline $15-200$ & Muy profundo & $17-20$ & 2 \\
\hline$>201$ & Extremadamente profundo & $<21$ & 1 \\
\hline
\end{tabular}

\subsection{Textura del suelo}

El tamaño y tipo de separado promedio en el perfil del suelo determina la capacidad de infiltración y de su permeabilidad. Esta propiedad establece la capacidad del suelo para retener o liberar elementos a las fuentes de agua, ya sea por la escorrentía o por las aguas de drenaje interno. 
Tabla 9. Clasificación y características de la textura del suelo según el porcentaje de los separados y en especial la clase textural

\begin{tabular}{|c|c|c|c|}
\hline \multicolumn{3}{|c|}{ Clasificación textural } & \multirow{2}{*}{$\begin{array}{c}\text { Valor como } \\
\text { Vulnerabilidad }\end{array}$} \\
\hline Según tamaño & Símbolo & $\begin{array}{l}\text { Clase textural } \\
\text { Denominación }\end{array}$ & \\
\hline \multirow{2}{*}{ Gruesa } & A & Arenoso & \multirow{2}{*}{10} \\
\hline & $\mathrm{AF}$ & Arenoso franco & \\
\hline Moderadamente gruesa & FA & Franco arenoso & 8 \\
\hline \multirow{3}{*}{ Medias } & $\mathrm{F}$ & Franco & \multirow{3}{*}{6} \\
\hline & $\mathrm{FL}$ & Franco limoso & \\
\hline & $\mathrm{L}$ & Limoso & \\
\hline \multirow{3}{*}{ Moderadamente fina } & F ArA & Franco-arcillo-arenoso & \multirow{3}{*}{4} \\
\hline & $\mathrm{FAr}$ & Franco arcilloso & \\
\hline & F ArL & Franco-arcillo-limoso & \\
\hline \multirow{3}{*}{ Finas } & ArA & Arcillo arenoso & \multirow{3}{*}{2} \\
\hline & $\operatorname{ArL}$ & Arcillo limoso & \\
\hline & $\mathrm{Ar}$ & Arcilloso & \\
\hline
\end{tabular}

\subsection{Contenido de materia orgánica del suelo}

Con base en lo reportado sobre los valores del contenido de materia orgánica del suelo según el clima, se propone como valores de vulnerabilidad al evento de la aplicación de plaguicidas en los cultivos, los cuales se supone que pasan al suelo, donde su posible retención puede ser generada por la materia orgánica del suelo.

\subsection{Porosidad del suelo}

Su vulnerabilidad al efecto de la aplicación de plaguicidas en los cultivos de una cuenca radica en lo siguiente: a menor densidad aparente, se genera mayor porosidad y el suelo tiene mayor capacidad de retener agua y, por lo tanto, se debe presentar menor escorrentía. Lo contrario se manifiesta con una alta
Tabla 10. Contenido de materia orgánica de suelo y su relación como amenaza en la contaminación de las aguas para consumo humano

\begin{tabular}{|c|c|c|}
\hline \multirow{3}{*}{ Según el clima } & $\begin{array}{c}\text { Materia } \\
\text { orgánica } \\
\%\end{array}$ & $\begin{array}{c}\text { Valor como } \\
\text { vulnerabilidad }\end{array}$ \\
\hline \multirow{3}{*}{ Frío } & $>10$ & 2 \\
\cline { 2 - 3 } & $10-5$ & 6 \\
\cline { 2 - 3 } Templado & $<5$ & 10 \\
\hline \multirow{3}{*}{ Cálido } & $10-5$ & 2 \\
\cline { 2 - 3 } & $5-3$ & 6 \\
\cline { 2 - 3 } & $3-2$ & 10 \\
\cline { 2 - 3 } & $5-3$ & 2 \\
\cline { 2 - 3 } & $3-2$ & 6 \\
\hline
\end{tabular}

densidad aparente; su condición extrema es cuando el suelo está compactado. La porosidad y su clasificación se toman de Montenegro y Malagón (1990). 
Tabla 11. Porosidad total del suelo y su relación con su vulnerabilidad al aporte de agua y sus componentes a las fuentes de agua de una cuenca hidrográfica

\begin{tabular}{|c|c|c|}
\hline $\begin{array}{c}\text { Porosidad total } \\
(\%)\end{array}$ & Clasificación & $\begin{array}{c}\text { Valor como } \\
\text { vulnerabilidad }\end{array}$ \\
\hline$>70$ & Excesiva & 2 \\
\hline $55-70$ & Excelente & 4 \\
\hline $50-55$ & Satisfactoria & 6 \\
\hline $40-50$ & Baja & 8 \\
\hline$<40$ & Muy baja & 10 \\
\hline
\end{tabular}

\subsection{Gradiente o inclinación de la vertiente}

Es la inclinación que presenta la superficie del terreno con respecto a un plano imaginario horizontal; generalmente, se expresa en porcentaje. Se parte de la base de que a mayor grado de la pendiente, mayor es la susceptibilidad del terreno para aportarle aguas a la red de drenaje de la cuenca.

\subsection{Longitud de la vertiente}

Corresponde a la longitud media de las laderas que bordean la red de drenaje, donde a menor longitud, mayor disponibilidad de agua en la fuente de la red de drenaje.

Tabla 12. Denominación de los grupos de pendiente

\begin{tabular}{|c|c|c|c|}
\hline Grupos & Pendiente $\%$ & Denominación & $\begin{array}{c}\text { Valor como } \\
\text { vulnerabilidad }\end{array}$ \\
\hline Plano & A1: $0-1$ & Plano (p) & 2 \\
(A) $0-3 \%$ & A2: $1-3$ & Ligeramente plano (Ip) & \\
\hline Inclinado & B1: $3-7$ & Suavemente inclinado (si) & 4 \\
(B) $3-25 \%$ & B3: $12-25$ & Moderamente inclinado (mi) & \\
\hline & C1: $3-7$ & Fuertemente inclinado (fi) & 6 \\
Ondulado & C2: $7-12$ & Suavemente ondulado (so) & \\
(C) $3-25 \%$ & C3: $12-25$ & Fueramente ondulado (mo) & \\
\hline & D1: $7-12$ & Suavemente quebrado (sq) & 8 \\
Quebrado & D2: $12-25$ & Moderamente quebrado (mq) & \\
(D) $25-50 \%$ & D3: $25-50$ & Fuertemente quebrado (fq) & \\
\hline Escarpado & E1: $50-75$ & Moderamente escarpado (me) & \\
(E) $>50 \%$ & E2: $>75$ & Fuertemente escarpado (fe) & \\
\hline
\end{tabular}

Tabla 13. Denominación de la longitud de la pendiente y su relación con la vulnerabilidad

\begin{tabular}{|c|c|c|}
\hline Denominación & $\begin{array}{c}\text { Longitud } \\
\mathrm{m}\end{array}$ & $\begin{array}{c}\text { Valor como } \\
\text { vulnerabilidad }\end{array}$ \\
\hline Corta & $<50$ & 8 \\
\hline Media & $50-150$ & 6 \\
\hline Larga & $150-300$ & 4 \\
\hline Muy larga & $>300$ & 2 \\
\hline
\end{tabular}




\subsection{Forma de la vertiente}

Permite la acumulación o liberación de agua en la vertiente, donde la plana y convexa tienden a presentar un aporte mayor de agua a la red de drenaje.

Tabla 14. Denominación de la forma de la pendiente y su relación con la vulnerabilidad

\begin{tabular}{|c|c|}
\hline $\begin{array}{c}\text { Denominación de las } \\
\text { formas }\end{array}$ & Valor como vulnerabilidad \\
\hline Rectilínea o plana & 6 \\
\hline Convexa & 4 \\
\hline Cóncava & 2 \\
\hline
\end{tabular}

\subsection{Ubicación del cultivo en relación con el sentido de la pendiente del terreno}

Si los cultivos se disponen en surcos, eras o camas, cuya dimensión mayor está orientada en el sentido de la pendiente del terreno, se facilita el transporte del plaguicida a las fuentes de agua. Este efecto se reduce si los cultivos se ubican en las curvas de nivel del terreno, porque esto reduce la velocidad de escorrentía cada vez que pasa entre dos surcos consecutivos.

Tabla 15. Ubicación del cultivo en relación con la pendiente del terreno y su vulnerabilidad en el escurrimiento de sus aguas

\begin{tabular}{|c|c|}
\hline Inclinación del surco de cultivo & $\begin{array}{c}\text { Valor como } \\
\text { vulnerabilidad }\end{array}$ \\
\hline En curva de nivel & 4 \\
\hline$<20^{\circ}$ de inclinación & 6 \\
\hline $20^{\circ}-40^{\circ}$ de inclinación & 8 \\
\hline $\begin{array}{c}\text { En sentido de la pendiente } \\
\text { del terreno }\end{array}$ & 10 \\
\hline
\end{tabular}

Escuela de Ingeniería de Antioquia

\section{RESULTADOS}

\subsection{Resultados de la estimación de la presencia del Metamidofos}

En la tabla 16 se tienen los resultados de los análisis de Metamidofos en los diferentes sitios muestreados en el agua, suelo y material vegetal (Ramírez y Zuluaga, 2007).

En las muestras de suelo analizadas no se encontró la presencia del Metamidofos, ya que es un compuesto que en el suelo se degrada fácilmente debido a su baja persistencia y alta movilidad, que le origina su lixiviación.

Según el Codex Alimentarius (FAO-OMS, 1963), conjunto de normas alimentarias internacionales encargado de establecer los límites permisibles de plaguicidas en alimentos, sean elaborados, semielaborados o crudos, se tiene que para las especies del género Brassica el límite permisible de Metamidofos es de $0,5 \mathrm{mg}$ por $\mathrm{kg}$ de producto vegetal. Para este caso, se detectó un valor de $5,1 \mathrm{mg} / \mathrm{kg}$.

Las variables que más incidieron en la alta presencia de Metamidofos son la época, frecuencia y dosis de aplicación del producto, lo cual concuerda con los resultados encontrados en las muestras de agua analizadas, en las cuales se pudo detectar la presencia de Metamidofos en los siguientes puntos: en la parcela de repollo (Brassica oleracea), en la fuente de agua a la salida de la parcela de repollo, en la parcela de remolacha, en la salida de la parcela de remolacha y en la quebrada antes de la bocatoma.

La muestra de agua que más presencia de Metamidofos arrojó fue la tomada en la parcela de repollo (Brassica oleracea), con una concentración de 60,350 $\mu \mathrm{g} / \mathrm{L}$, esto ocurrió debido a la alta frecuencia de aplicación del producto, a la práctica cultural de aplicarlo cuando se presenta lluvia, la cual facilita el transporte del producto hacia las partes inferiores del terreno, vertiendo a la fuente de agua. 
Tabla 16. Concentración de Metamidofos en planta, suelo y aguas de la cuenca hidrográfica de la Quebrada EI Salto

\begin{tabular}{|c|c|c|c|}
\hline \multicolumn{4}{|c|}{ Como contaminante del agua antes de la bocatoma del acueducto } \\
\hline \multicolumn{2}{|c|}{$\begin{array}{l}\text { Periodo de alta frecuencia de lluvias. } \\
\text { Cultivo: Repollo (B. oleracea) }\end{array}$} & \multicolumn{2}{|c|}{$\begin{array}{l}\text { Periodo de baja frecuencia de lluvias } \\
\text { Cultivo: Remolacha (Beta vulgaris) }\end{array}$} \\
\hline Zona de muestreo & $\begin{array}{c}\text { Concentración de } \\
\text { Metamidofos } \\
\mu \mathrm{g} / \mathrm{L}\end{array}$ & Zona de muestreo & $\begin{array}{c}\text { Concentración de } \\
\text { Metamidofos } \\
\mu \mathrm{g} / \mathrm{L}\end{array}$ \\
\hline Tanque de escorrentía & 60,350 & En el tanque de escorrentía & 0,04 \\
\hline $\begin{array}{l}\text { Fuente de agua aledaña } \\
\text { a la parcela }\end{array}$ & 0,020 & $\begin{array}{c}\text { Fuente de agua aledaña a la } \\
\text { parcela }\end{array}$ & 0,129 \\
\hline $\begin{array}{c}\text { Antes de la entrada del agua } \\
\text { a la bocatoma }\end{array}$ & 0,351 & $\begin{array}{c}\text { Antes de la entrada del agua } \\
\text { a la bocatoma }\end{array}$ & $<0,010$ \\
\hline \multicolumn{4}{|c|}{ Como contaminante del agua en la red de distribución del acueducto } \\
\hline \multicolumn{3}{|c|}{ Concentración de Metamidofos $\mu \mathrm{g} / \mathrm{L}$} & $<0,010$ \\
\hline \multicolumn{4}{|c|}{ Como contaminante en los suelos de la cuenca $(\mathrm{mg} / \mathrm{kg})$} \\
\hline \multicolumn{3}{|c|}{ En el suelo de la parcela con repollo (Brassica oleracea) } & No se detectó \\
\hline \multicolumn{3}{|c|}{ En el suelo de la parcela con remolacha (Beta vulgaris) } & No se detectó \\
\hline \multicolumn{4}{|c|}{ Como contaminante en material vegetal $(\mathrm{mg} / \mathrm{kg})$} \\
\hline \multicolumn{3}{|c|}{ En la planta de repollo (Brassica oleracea) } & 5,1 \\
\hline
\end{tabular}

Las muestras tomadas en el tanque de la bocatoma y en la red de distribución del acueducto municipal arrojaron datos menores que $0,010 \mu \mathrm{g} / \mathrm{L}$.

\subsection{Lombriz de tierra como indicador biológico de contaminación}

En la tabla 17, se tiene el número de lombrices por $\mathrm{m}^{2}$ en dos parcelas muestreadas. Al respecto, se encontró que existe una diferencia muy marcada en las dos parcelas muestreadas, destacándose el bajo número de individuos en el lote donde se tiene el cultivo de repollo (Brassica oleracea), con aplicación de Metamidofos.
Tabla 17. Lombrices (Lumbricus terrestris) en el suelo de la parcela experimental. Área de muestreo: $0,25 \mathrm{~m}^{2}$. Profundidad: $0,1 \mathrm{~m}$

\begin{tabular}{|c|c|}
\hline Parcela muestreada & $\begin{array}{c}\text { Número de } \\
\text { lombrices } \\
\mathrm{m}^{2}\end{array}$ \\
\hline $\begin{array}{c}\text { Cultivo de repollo con aplicación } \\
\text { de Metamidofos }\end{array}$ & 36 \\
\hline $\begin{array}{c}\text { Parcela con pasto kikuyo } \\
\text { (Pennisetum clandestinum L.) }\end{array}$ & 424 \\
\hline
\end{tabular}

\section{Estimación del riesgo}

Con base en la propuesta de Gómez (2006a), con la cual se podría evaluar el riesgo por la 
contaminación de Metamidofos en la cuenca hidrográfica de la quebrada El Salto del municipio de El Santuario, se obtienen los resultados que se expresan en la tabla 18.

\subsection{Evaluación del valor del riesgo}

El valor del riesgo resulta del producto entre la suma de las amenazas por la suma de la vulnerabilidad de la cuenca, por el cual se clasifica según la información de la tabla 19.

Tabla 18. Valor del riesgo por la contaminación de Metamidofos en la cuenca de la quebrada El Salto

\begin{tabular}{|c|c|c|}
\hline No. & AMENAZA (A) & Valor como amenaza \\
\hline 1 & Según la toxicidad del plaguicida & 10 \\
\hline 2 & Según la solubilidad del plaguicida & 10 \\
\hline 3 & Evaluación de la precipitación & 8 \\
\hline 4 & Evaluación de la frecuencia de la lluvia & 2 \\
\hline 5 & Evaluación de la duración de la lluvia & 38 \\
\hline & Total de la amenaza (A) & Valor como vulnerabilidad \\
\hline No. & VULNERABILIDAD (V) & 10 \\
\hline 1 & Índice de forma & 8 \\
\hline 2 & Tiempo de concentración & 10 \\
\hline 3 & Profundidad efectiva del suelo & 10 \\
\hline 4 & Textura del suelo & 4 \\
\hline 5 & Contenido de materia orgánica del suelo & 8 \\
\hline 6 & Densidad aparente del suelo & 6 \\
\hline 7 & Gradiente o inclinación de la vertiente & 4 \\
\hline 8 & Longitud de la vertiente & 6 \\
\hline 9 & Forma de la vertiente & 74 \\
\hline 10 & Ubicación de los cultivos en relación con el sentido de la & 38 * $74=2812$ \\
\hline & pendiente del terreno & \\
\hline & VALOR DEL RIESGO = A x V la vulnerabilidad (V) & \\
\hline
\end{tabular}

Tabla 19. Estimación del riesgo como resultado de la aplicación de plaguicidas en la cuenca hidrográfica que surte el agua para consumo humano (Tomada de Gómez, 2006a)

\begin{tabular}{|c|c|}
\hline Valor del riesgo & Categoría del riesgo \\
\hline$<200$ & Muy bajo \\
\hline $201-800$ & Bajo \\
\hline $801-1800$ & Moderadamente bajo \\
\hline $1801-3200$ & Alto \\
\hline $3201-5000$ & Muy alto \\
\hline
\end{tabular}

El resultado obtenido para dicha calificación fue de 2812, valor en el rango alto, lo que indica que es relevante. Las variables consideradas para calcular el riesgo y la vulnerabilidad se manifiestan debido a que los agricultores del área de estudio tienen los cultivos cerca de la fuente de agua, lo que unido a la poca práctica de conservación de suelos, el uso indiscriminado de plaguicidas, la baja educación técnica y ambiental en el manejo seguro de biocidas determinan algunos de los principales factores que inciden directamente en que se 
presente un riesgo de contaminación de las aguas para consumo humano.

Las variables que se pueden mitigar para esta investigación, al evaluar el riesgo de contaminación por plaguicidas en aguas para consumo humanos son:

Componentes de amenaza

Toxicidad del plaguicida

Componentes de la vulnerabilidad

Contenido de materia orgánica del suelo

Gradiente o inclinación de la vertiente

Ubicación de los cultivos en relación con la pendiente del terreno

\section{CONCLUSIONES}

Se estimó la concentración del Metamidofos en el repollo (Brassica oleraceae), en el suelo y en el agua, presentándose trazas del producto en el material vegetal, en el agua de escorrentía, en 3 sitios del flujo de agua de la quebrada El Salto, ubicados desde la salida de la parcela de escorrentía hasta la sección que deriva a la bocatoma del acueducto municipal.

No se detectó la presencia del producto en el suelo, en la bocatoma ni en la red de distribución del acueducto municipal.

Dentro de las variables ambientales evaluadas se determinó que el manejo de suelos es uno de los factores que más influye en el transporte y comportamiento del producto en la cuenca.

Los indicadores evaluados, como la escorrentía, el coeficiente de escorrentía y la pérdida de suelo arrojaron una presencia más significativa del contaminante en la época de alta frecuencia de lluvias.

El manejo y aplicación del Metamidofos en la zona de estudio generó una alta incidencia en el riesgo de contaminación calculado por este producto, en la microcuenca.

El potencial del riesgo por la contaminación de este insecticida se manifestó en la reducción de la población de la lombriz de tierra (Lumbricus terrestris).

De acuerdo con la metodología aplicada para la valoración del riesgo de contaminación por Metamidofos en la microcuenca El Salto, se encontró nivel de riesgo alto, representado en la alta incidencia en el cálculo de las variables de amenaza y vulnerabilidad evaluadas.

\section{RECOMENDACIONES}

Para analizar plaguicidas se hace necesario que la toma de muestras se cumpla cuando estén aplicándolos y que se envíen de inmediato al laboratorio, debido a la rápida degradación y movilidad de algunos plaguicidas.

La aplicación de los plaguicidas en una zona se deben fundamentar en la presencia del organismo y en especial en el nivel de su incidencia en el cultivo.

Para el control de plagas, se deben generar opciones menos agresivas al medio ambiente, como otros productos o la reducción en la frecuencia de aplicación.

Hay que realizar estudios de identificación de plagas y enfermedades donde se conozcan los ciclos de vida, cómo es su reproducción, dónde habitan, de qué se alimentan con el fin de hacer seguimiento e implementar las técnicas del MIPE (manejo integrado de plagas y enfermedades) por medio del control cultural, biológico y físico.

Se deben respetar los retiros a las fuentes hídricas como lo establecen las leyes y decretos reglamentarios, para este caso se necesita identificar los dueños de los predios y pactar con ellos el respeto de los retiros con el objeto de mitigar el impacto de estos plaguicidas a los diferentes ecosistemas. 
El programa de extensión que se aplique para reducir la concentración de un plaguicida en una fuente de agua y, en especial, si esta es utilizada para las diferentes actividades humanas, debe estar orientado al manejo de las variables que reducen la concentración del producto en las aguas, como son: disminución de las dosis del plaguicida, uso de opciones con productos menos contaminantes y ante todo implementar estrategias conservacionistas del suelo, que tiendan a reducir el coeficiente de escorrentía y que incrementen el tiempo de concentración de las aguas que provienen de cada unidad de producción de la cuenca que se analiza.

Es útil realizar dentro de la comunidad que habita la cuenca hidrográfica campañas y talleres de educación ambiental para informarles el riesgo que corren los habitantes de la cuenca y la comunidad aguas abajo, para que tiendan a un manejo adecuado de plaguicidas, con el objetivo de reducir el impacto en el ambiente y en la salud humana, e igualmente se conozcan las alternativas agroecológicas para disminuir dicho impacto, tales como siembra siguiendo las curvas de nivel o a través de la pendiente, establecimiento de barreras vivas con especies forrajeras y alelopáticas, labranza mínima, aplicación de abonos verdes y la implementación de parcelas de escorrentía cerrada.

Para mitigar el componente de amenaza relacionado con la toxicidad del plaguicida se hace necesario cambiar la aplicación a productos de menor categoría toxicológica.

\section{BIBLIOGRAFIA}

ARANGO, M. C. Módulos de capacitación sobre plaguicidas. Universidad Católica de Oriente. Unidad de Gestión Ambiental, 1996. 28 p.

COLOMBIA. MINISTERIO DE SALUD. Decreto 1843 de 1991. Uso y manejo de plaguicidas. Santafé de Bogotá, $72 \mathrm{p}$.

CORPORACIÓN AUTÓNOMA REGIONAL RIONEGRO NARE (Cornare). Lineamientos generales para abordar la problemática del uso y manejo de plaguicidas y propuestas de solución en la jurisdicción de Cornare, 2003. 65 p.
ESPEJO, G. J. Reglamentación de corrientes en la subcuenca del río Tona. En: Memorias del Tercer Congreso Colombiano de Cuencas Hidrográficas CVC, 1985. $40 \mathrm{p}$.

ESPINAL, T. Zonas de vida o formaciones vegetales de Colombia. Memoria explicativa sobre el mapa ecológico. Bogotá. Instituto Geográfico Agustín Codazzi, 1977. $38 \mathrm{p}$.

FAO-OMS. Límites máximos del Codex para residuos de plaguicidas en alimentos. 1963 [online]. [Citado el 19 de febrero de 2007]. Colombia. http:// www.codexalimentarius.net $/ \mathrm{mrls} /$ pestdes/pest_ref-5html-E10E2

GALEANO, L.; TABARES, M. y BOLÍVAR, J. Identificación de factores de riesgo por el uso y manejo de plaguicidas en las cuencas que abastecen los acueductos de las cabeceras municipales: Antioquia 1999-2001. Dirección Seccional de Salud del Departamento de Antioquia, 2001. 450 p.

GIRALDO, P.; HERNÁNDEZ, J.; HURTADO, L. y SUÁREZ, L. Diagnóstico sobre uso y manejo de plaguicidas a nivel de expendedores de los municipios de Marinilla, El Santuario, Rionegro y El Carmen de Viboral, 2002. $50 \mathrm{p}$.

GÓMEZ, F. E. Construcción, implementación y manejo de parcelas de escorrentía. Universidad Nacional de Colombia, Facultad de Ciencias, Medellín, 1985. 42 p.

GÓMEZ, F. E. Procesos erosivos: Estrategias para su caracterización e implementación de sus prácticas básicas de control y prevención. Universidad Nacional de Colombia. Facultad de Ciencias. Medellín, 1999. 154 p.

GÓMEZ, F. E. Manual tutorial: Manejo y conservación de suelos. E. Gómez. Medellín, 2006a. 123 p.

HERNÁNDEZ, H. Producción limpia rural subsector plaguicidas y su impacto sobre el recurso suelo. Corporación Autónoma Regional del Valle del Cauca (CVC). En: Seminario Nacional de Suelos, 2001. 20 p.

INSTITUTO COLOMBIANO DE GEOLOGÍA Y MINERÍA INGEOMINAS. Estado del conocimiento. Proyecto: Investigación y zonificación de amenazas por procesos de aprovechamiento del subsuelo. Informe final. Bogotá - Colombia, diciembre de 2002. [Online] [Citado el 26 de diciembre de 2006]. Colombia. http://productos. ingeominas.gov.co/productos/OFICIAL/geoamena/informes/EstadConocimiento.pdf.

LÓPEZ, F. R. Degradación del suelo: Causas, procesos, evaluación e investigación. Centro Interamericano de Desarrollo e Investigación Ambiental y Territorial. Universidad de los Andes. Mérida, Venezuela, 2000. $259 \mathrm{p}$. 
LOZANO, L. M. Intoxicación aguda por Metamidofos en trabajadores de cultivos de hortalizas. [Online]. [Citada el 31 de octubre de 2006]. Nicaragua. http//: www. cuencarural.com/servicios/seguridad_e_higiene_rural/intoxicación_aguda_por_metamidofos_entrabajadores_de_hortalizas/.htm

MONTENEGRO, G. H. y MALAGON, C. D. Propiedades físicas de los suelos. Bogotá, IGAC, 1990. 800 p.

ORGANIZACIÓN DE LAS NACIONES UNIDAS PARA LA AGRICULTURA Y LA ALIMENTACION FAO. Hydrological techniques for upstream conservation. Roma. Conservation Guide No. 2, 1976. 134 p.

PROINGECON. Estudio hidrológico, manchas de inundación y propuesta de reglamentación de las quebra- das Bodegas, La Tenería, El Salto y Portachuelo de la cabecera Municipal de El Santuario. Secretaría de Planeación, 2000. 134 p.

RAMÍREZ, D. y ZULUAGA, A. M. Evaluación del riesgo de contaminación por Metamidofos en la microcuenca El Salto del Municipio de El Santuario, Antioquia. Rionegro, 2007. 95 p. Trabajo de grado (Ingeniería Ambiental). Universidad Católica de Oriente. Facultad de Ingeniería.

SMITH, R.; VELEZ, J.; CABALLERO, H.; CASTIBLANCO, C.; RAMÍREZ, C.; ARANGO, A.; RAVE, C.; ÁLVAREZ, A.; BOTERO, V. y ESCOBAR, D. Evaluación de la vulnerabilidad ante amenazas naturales en cuencas hidrográficas. Boletín Ciencias de la Tierra No. 17. 2005. p 35-62. 